\title{
Methodologies and applications in Digital China
}

\author{
WENG JingNong ${ }^{*}$, RAFIQUE Irfan \& ABBASI Maissom Qanber \\ School of Computer Science and Engineering, Beihang University, Beijing 100191, China
}

Received June 25, 2012; accepted August 7, 2012

This article portrays a concise review on an extensive range of state-of-art advancement in methodologies and applications for Digital China, which includes models, algorithms, theory framework, engineering technology, science methodology, practice applications, etc.

Digital China, spatial information and technology, new research advancement

Citation: $\quad$ Weng J N, Rafique I, Abbasi M Q. Methodologies and applications in Digital China. Chin Sci Bull, 2012, 57: 3761-3768, doi: 10.1007/s11434-012-5446-y

Digital China can be viewed as a nationwide strategic project for China in response to the challenge of sustainable development of population, resources, and environment. There have been significant advancements in areas like Digital Earth (DE) concept and frameworks, spatial data modeling, storage and processing, information processing technologies for DE, emerging technologies for display and visualization, spatial computing, spatial navigation, etc. Also progress has been achieved in employing contemporary web technologies in conjunction with DE application. The paper also summarizes other recent research progress made towards the ultimate objective of Digital China.

\section{Geospatial data modeling and storage}

The volumes of global geospatial information, generated by various geospatial applications and collected by newly designed acquisition devices are growing enormously. Efficiently organizing and effectively integrating global multi-source geospatial information thus becomes a challenging task. Gong et al. [1] have proposed a global pyramid data model, which organizes all geospatial data as the structure of a global multi-resolution pyramid. A unique identification is assigned to each pyramid tile, which codes the twodimensional value of latitude and longitude as one-dimen-

\footnotetext{
*Corresponding author (email: wengjn@ @uaa.edu.cn)
}

sional Morton code, as well as contains the information of data resolution and time phase. The authors have further proposed integration framework of virtual globes and geospatial web services. This results in analysis enabled virtual Globes offering sharing and visualization of multi-source geospatial data.

Xia et al. [2] have introduced an approach for the organization and storage of spatial data. Their approach is based on the Laplacian pyramid framework. In the proposed approach biorthogonal transformation is utilized to split high and low frequency data, to build a spatial index for the transformed frequency data. This methodology economizes storage requirements and network overloads for progressively transmitting spatial image.

Zhao et al. [3] have proposed a Global Remote Sensing Data Hierarchical Model (GRHM). The model relies on the image pyramid and tiling techniques. GRHM is independent of physical storage. A physical structure for storage is proposed and multi-dimensional remote sensing data are deployed on web servers to support lightweight web map services (WMSs). In order to enhance efficiency of WMSs, a data de-clustering approach, utilizing Hilbert space filling curve, is used for the distributed storage. For web browsers, the authors have presented an Open Geospatial Consortium WMS and a web map system. The performance of the proposed WMSs is proven by conducting experiments on real remote sensing datasets.

Rapid development in satellite observation systems has 
brought about a massive increase in remote sensing data. This calls for an efficient storage and management of such data. Realizing this fact, Lü et al. [4] took 13 data storage centers, including NASA EOS, Google Earth, GeoEye, China Centre for Resources Satellite Data and Application (CCRSDA), etc., as practical examples, and analyzed their characteristics and main advantages from storage and architecture perspectives. The research is a comprehensive guideline towards establishing global remote sensing data storage model and making it more suitable for ever growing remotely sensed data.

\section{Digital city modeling and applications}

The digital city has been transformed from a novel concept to a practical and effective means of supporting urban planning and management. 3D digital city is one of the most important applications with a wide range of usages. At the same time, the advent of modern web service technologies allows the possibility of 3D geospatial data to be distributed on the web. Yang et al. [5] have discussed the feasibility of representing 3D city building models as a kind of data resource service on the web which could also coexist with various web applications. Their approach to model 3D city buildings follows hierarchical representation based on structural details. The proposed approach has a vast potential in urban planning, architectural design, tourism, etc. Currently the study has been proven to be successful in a real state application in Shenzhen, China.

Where GIS and digital urban technologies are developing rapidly, exploring the urban villages' characteristics via theoretical analysis and quantitative methodologies is a big concern. Tang et al. [6] first compared different digital city concepts, examined common aspects of digital city definitions and proposed an urban digital operating system (Urban DOS) that would be useful to improve life quality, socioeconomic functions and sustainable development in a city and its surrounding areas. They have established the technical basis for the Urban DOS in the intersection between technology-oriented products (TOPs) and customized application products (CAPs), and developed a procedure for designing a framework for a digital city based on Urban DOS with TOPs and CAPs. They also have demonstrated the initial development of Urban DOS for Lijiang City in China to explain their digital city concepts and applications.

\section{Spatial modeling frameworks}

Spatial modeling helps urban and rural planning from various perspectives, including distribution of people and activities in different areas of region, economic forecast, environmental strategies, etc. Tong et al. [7] have proposed a mathematical model based on spatial analysis for categori- zation and characterization of urban villages. The case study, employing ArcGIS tools, distance and density calculation, and socioeconomic and spatial attributes conjunction, has been used for a total of 89 villages in Shenzhen Special Economic Zone, and identifies urban villages' 31 spatial variables in social, economic, location and physical aspects. The proposed strategy can be beneficial to summarizing most important characteristics and differences among urban villages and providing improvements in their management and guidance policies.

For improving image quality in remote sensing applications, Zhang et al. [8] have proposed a fusion algorithm employing non-subsampled Laplacian pyramid (NLP) and bi-dimensional empirical model decomposition (BEMD). The proposed methodology avoids fusion of detail information on different scales, resulting in decreased scalealiasing. Their experimental results show that the proposed scheme improves the spectral and spatial detail quality as compared to other algorithms such as intensity-hue-saturation (IHS) transformation, discrete wavelet transform (DHT) and a'-Trous wavelet.

Realizing the importance of modeling framework for integration of models for hydrology and land surface process, Nan et al. [9] have proposed the integrated modeling environment (IME) for Heihe River Basin in an extendable, efficient and easy to use way. Further, while discussing the limitations of the existing methodologies, they discussed the challenges that their proposed model addresses in terms of intelligence and robustness. Through two groups of experiments, the authors also showed the effectiveness, reliability and practicality of the proposed modeling environment based model integration.

Cao et al. [10] have explored the influence of small-scale water conservancy on flood forecasting precision. In doing so, they proposed an approach to modeling the detention of floods using small reservoir. The proposed approach was applied to the data available via American land resources satellite named Landsat TM/ETM+. The authors demonstrated the usefulness of their approach with a case study, where the flood of August 2008 was taken as an example.

In the research of tectonic movement and different geodynamic processes for disaster prevention and reduction, the structure of global lithosphere is very vital. In this regard, Yu et al. [11] have proposed a three-dimensional (3D) modeling and visualization framework for global lithosphere by utilizing Spheroid Degenerated-Octree Grid. Their approach is novel in that it not only overcomes the shortcomings in the related research but also highlights the lithosphere features with natural and realistic details.

\section{Map visualization technologies and applications}

In geospatial information, map visualization is one of the 
prime concerns. Thematic maps are designed to demonstrate particular features of concepts. They function as an indispensable tool in geographic research. Keeping in mind the importance of thematic mapping in geographical research, Qi et al. [12], using two approaches namely phase-to-phase and multi-aim scheme balance modes, have developed an integrated solution to selection, optimization and use of mathematical models for thematic map layer production, editing, knowledge innovation and product making. The authors described a case study for eco-environment fragility and sensitivity assessment mapping in Beibuwan (Guangxi) District in China. Case studies utilizing dynamic system method for the model optimization for ecological security adjustment mapping (in Xishuang Banna, Yunnan Province) and multi-phase mode in the models for forest fire and infectious diseases mapping were also described.

Chen et al. [13] have presented an algorithm for reconstructing a contour map from raster Digital Elevation Model (DEM) data for DE and other terrain platforms in real time. With the power of programmable pipelines, they made the elevation gradient map out of original terrain vertex data, and the final contour lines with image-space processing. The method was implemented on 3D DE System, and tested on different PC platforms. The authors also demonstrated the efficiency of their proposed approach.

From map visualization point of view, metro maps are well known for illustrating and visualizing abstract information of transportation networks. Focusing on generating quality metro maps, Dong et al. [14] proposed a set of principles for metro maps reliability and aesthetic considerations. A dynamic segmentation based methodology is proposed for automatic maps generation. Furthermore, cartographic methods for generalization of physical attributes such as shape, angle, topology etc., are used for the map routes. The approach has been applied on Beijing metro plan map indicating the effectiveness of the proposed methodology in producing simpler and clearer maps which are useful for travelers.

In the domain of land use mapping, remotely sensed images have now become a very powerful source. Gao et al. [15] have explored the differences between image-derived land use maps and traditional maps by quantifying intellectual and graphical map complexities, where the former is concerned with meaningful information on the map and the later addresses the spatial characteristics of graphical content of the map. The approach was applied to two maps from Jiufeng in China. The results demonstrated that the quantification of intellectual and graphical complexities was a sensible way to evaluate the performance and effectiveness of land use map.

\section{Geospatial change detection}

For better management and utilization of resources, timely and accurate change detection of earth's feature is vital. Sun et al. [16], realizing the sensitivity of image radiometric features to change detection, have proposed radiometric processing before change detection to make radiometric features of multi-temporal images consistent. They introduced a preprocessing approach to radiometric consistency, utilizing wavelet transformation and a spatial low-pass filtering. This approach first separates the high frequency information and low frequency information by wavelet transform. The processing of relative radiometric consistency based on a low-pass filter is then conducted on the low frequency parts. Experiments have shown that this approach is effective when it is used for processing radiometric consistency during change detection, and can also be widely used in other ways.

Zhang et al. [17] have devised an approach toward object based change detection by first segmenting two different temporal images using the mean shift procedure to obtain corresponding objects and subsequently detecting change based on the integration of the corresponding objects' intensity and texture differences. Experiments were conducted on panchromatic images and multispectral images and results show that the integrated measure is robust with respect to noise and illumination changes.

The gradient based on landscape metrics analysis is being widely employed to study the landscape pattern changes due to the urbanization. Yang et al. [18] have discovered the trend of spatiotemporal changes in Beijing metropolitan area during the past 15 years, by computing several landscape metrics using a moving window along a 96-km-long transect across Beijing metropolitan area from west to east. The results not only show the spatial and temporal landscape characteristics of Beijing City hypothetical framework but also reveal some new patterns.

Zhou and Sun [19] discussed the impact of land use and land covers change (LUCC), as one of the most significant indicator of environment change that takes place due to various human activities on earth's environment. They modeled spatiotemporal pattern of land cover change using multi temporal satellite images. The proposed approach is not only capable of monitoring the extent and spatial pattern of urban expansion but also capable of revealing the origins of varying land use, leading to better counting for the cost of built-up area expansion. The case study area was the Pearl River Delta region in southern China. The results show that cities and towns have been sprawling in general, demonstrating two growth models that are closely related to the economic development stages.

\section{Virtual geographic visualization}

Virtual globe provides an intuitive and appealing way to visualize geospatial information, and is being used frequently to bring the globe-scale geospatial information to 
our fingertips. Weng et al. [20] have proposed a common core framework for a visual engine for efficient development of DE application. They have also discussed parametric model of the Earth, scheduling and optimization in visual field, choice of 3D graphics library, and designing component-based visual engine framework of DE, and relationship among these four components.

Yue et al. [21] put forward an approach for the establishment of an analysis-enhanced virtual globe based on the integration of virtual globe and interoperable geoprocessing services. This approach significantly enhances the use of geospatial data by making it much easier to publish, discover, access, process, integrate, and analyze earth science data and information from the distributed web sources, thus contributing to the development of DE.

Lü [22] highlighted the importance of geographical analysis in Virtual Geographic Environment (VGE) by systematically examining the contemporary researches in the related areas, such as spatial analysis and simulation. Dividing VGE into specific environments, their associated characteristics are studied. This research can be treated as a foundation for further directions in VGE domain.

\section{Earth observation satellites}

$\mathrm{DE}$ is a multi disciplinary field involving information technology, geosciences as well as space technology. It is also an evolutionary development of space era, characterized by earth observation satellites. Earth observation satellites are, thus, the key technologies for building a DE. Guo [23] highlighted the role of China in the development of Earth observing satellites. The author described and summarized the main characteristics of four satellite series, namely resource satellites, environment satellites, meteorological satellites and ocean satellites.

$\mathrm{Xu}$ [24] presented the land observation satellite system of China, describing the requirements for satellite payloads regarding temporal, spatial and spectral resolution aspects to establish a DE. The author further introduced applications of land satellites under the DE framework from different perspectives including special subject services, data support, and integrated information services and concluded that China's land observation satellites as well as ground processing systems would be the fundamental constituents of the DE system and would greatly promote applications of DE.

The autonomous rendezvous technology is vital for space military missions, future on-orbit service, space debris removal, and deep-space detection.

Relative navigation is a key technology for autonomous rendezvous, owing to the precision of the relative navigation, fuel economy, safety of trajectory, and mission success. Li et al. [25] researched angles-only relative navigation for autonomous rendezvous and proposed schemes for deter- mining degree of observability (DOO) and orbital maneuver's latent range information in order to analyze and enhance the precision of relative estimates of position and velocity. The corresponding equations have been developed considering optical camera as the only possible sensor for relative measurement. The Newton iterative method is used to obtain the expressions for the DOO of relative navigation. Numerical result shows the effectiveness of this approach to depict the observability level of relative position and velocity. Moreover the latent range information has been found useful to enhance the DOO of the angles-only relative navigation.

The optimization of the relative trajectory for autonomous rendezvous is essential for optimizing its performance. Li et al. [26] carried out a study on the optimal multi-objective trajectory design relying on the close-looped control for autonomous rendezvous. The authors' approach selects total velocity cost as well as relative state robustness of the closelooped control as the objective functions. The state and measurement equations for angles-only relative navigation between spacecrafts are developed based on relative dynamics equations. The authors employed linear covariance analysis to analyze the close-looped control covariance, and the relative position robustness's expressions. The relative velocity robustness was defined based on the covariance results. This multi-impulsive rendezvous problem was subsequently solved using multi-objective optimization algorithm of NSGA-II. Monte Carlo simulation was then executed a hundred times to exhibit the validity of the objective functions and the covariance results.

\section{Information processing technologies for DE}

Information Processing Technologies for DE have gained considerable momentum during recent years for Digital China. Xiong et al. [27] have formulated a Semi-Supervised Classification (SSC) method describing each class' feature space by Gaussian Mixture Model (GMM). Experiments show that the proposed method can achieve high classification accuracy with small amount of labeled data compared to other similar methods.

Xiao et al. [28] have devised target space exteriorization sampling method (TSES) to generate pseudo absence data based on presence data directly in feature space for landslide susceptibility mapping. TSES externalizes a presence sample transforming it to a pseudo absence sample. This was done by swapping the value of one of its features with a new one outside this feature's value range for all presence data. This method was compared with two existing methods, buffer controlled sampling (BCS) and iteratively refined sampling (MS), in a study area of Shenzhen city, achieving promising results.

From the point of view of tourist attractions, Zhang et al. [29] discussed the practical significance of spatial distribu- 
tion of hyperlinks. In this regard, as part of the case study, three mountains of Anhui Province, China were chosen to evaluate correlation between spatial distributions of attraction's tourist source markets and their official website's inlinks and tourism indexes such as the spatial concentration index and the attraction radius (calculated by inlinks).

Considering the importance of extracting positional information from unstructured representation, Gong et al. [30] have proposed a strategy whereby they have generated locality descriptions and positioning localities in order to locate target object (TO) by using the information of the reference object (RO). The selection of the RO is determined by probability density function (PDF). For computing the PDF, the authors employed Euclidean distance and Voronoi stolen-area methodology. The effectiveness of the proposed method was also demonstrated using a case study.

Qiao et al. [31] have developed a unified conceptual model (UCM) from the vegetation-impervious surface-soil (VIS) model to make the extracting impervious surfaces from multi-source satellite imagery more effective and accurate. UCM utilizes the decision tree algorithm having indices of spectrum and texture, etc. In each bifurcation point of the tree, the corresponding index is selected for the extraction information by threshold segmentation method.

For characterizing the status of the land surface, soil moisture (SM) is one of the most significant physical attributes. Li et al. [32] simulated SM from 1951 to 2008 using station observations and constructed an atmospheric field (ObsFC) for the Community Land Model version 3.5 (CLM3.5). CLM 3.5 is an improved and updated version of community land module derived from the Common Land Model and the Land Surface Model (LSM) of the National Center for Atmospheric Research. ObsFC consists of four status variables including air temperature, wind speed, atmosphere pressure and specific humidity, and two flux variables including precipitation and radiation. The authors compared their simulated SM with the existing remote sensing data, observations and estimations made by various land models. In this way the authors proved the capability of their approach to reproduce temporospatial characteristics and long-term SM variation trends over China. The authors also indicated and explained various SM variation trends throughout China in different time periods. The overall spatial pattern of SM has been found to be progressively decreasing and varyingly distributed in terms of drymoist zones from the southeast to northwest of China.

Jian et al. [33] reported the creation of a model to retrieve soil moisture saturation based on multispectral remotely sensed data. Soil brightness and soil wetness, calculated from the tasseled cap transformation, were utilized to obtain soil moisture saturation. Using this model, a soil moisture saturation map of Maoergai District, in Sichuan Province of China, was created. The model was evaluated using vegetation distribution and soil type data from the year 2000. The result proved the viability of the model for soil moisture saturation. The authors also discovered that the vegetation type, vegetation distribution and soil type had strong correlation with soil moisture saturation.

Sheng et al. [34] have proposed a vehicle detection scheme which employs semantic analysis of traffic surveillance video using spatio-velocity statistical modeling. The proposed model utilizes trajectory clustering and semantic events detection. The approach has been tested empirically and the results prove the effectiveness of the proposed scheme.

In the domain of the distributed geospatial information processing, $\mathrm{Xu}$ et al. [35] have studied the estimation of the above-ground biomass (AGB) and carbon storage in arid and semiarid ecosystems. The $\mathrm{Mu}$ Us Desert in China was chosen as the case study and HJ-1 A/B satellite data together with field measurement data were used for estimation of shrub AGB and carbon storage. The results indicate that the vegetation indexes have higher correlations than the spectral reflectance.

Jiang et al. [36] have explored the advanced and practical means to monitor, recognize and locate forest fires, which are frequent natural disasters. The proposed approach leads to early detection and quick fighting of forest fires with realtime positioning. The methodology also includes measures such as increasing recognition ability on the basis of color and shape, and eliminating factors of disturbance.

Tian et al. [37] demonstrated a method for atmospheric correction while processing HJ-1 A/B CCD images at China coastal waters. In doing so, they employed moderate-resolution imaging spectroradiometer-Terra (MODIS-Terra) aerosol information. With the aid of this atmospheric correction approach, the water-leaving radiance over Chinese coastal water area has been derived successfully. The proposed scheme can be beneficial to the research and applications in the turbid coastal zones.

Jiang et al. [38], while analyzing the current limitations in the approaches to detect underground coals fires, suggested detecting coal fire temperature using Land sat 5 TM images and Generalized Single-Channel Algorithm (GCA). The authors also demonstrated the effectiveness and feasibility of the proposed method by applying it in Wuda coalfield, Inner Mongolia, China. Using their approach the temperature error can be relatively small. Considering coal fire burning being an environmental danger, this study is useful in context of estimating emissions of noxious gas as a result of coal fire.

Wang et al. [39] have introduced a spectral characteristics-based palaeochannel information retrieval. The model differentiates palaeochannels from active river channels based on analysis of spectral features and remote sensing mechanism. The model also helps to identify a palaeochannel in remotely sensed image. This model follows the process of supervised classification followed by farmland masking and primary component analysis. A subsequent underground palaeochannel information extraction follows 
this course and finally information is integrated to create palaeochannel system. Their study, based on the proposed approach, suggests that the dense vegetation areas in Eastern China are distinct from current river channels in terms of spatial characteristics and palaeochannels spectrum.

Zhang et al. [40] have proposed a method for near field image detection and reconstruction. The authors argued that the existing Fourier method did not support the expression of the closed-form nature, and hence cannot be employed for the near-field scenarios. The proposed approach is numerically based and yields efficient and accurate results. Experiments and simulations have further confirmed the viability of the proposed solution.

Zhang et al. [41] utilized water assessment tool (SWAT) to study effects of human activities and climate changes on runoff of the rivers. The authors selected Huifa River Basin in Northeast China as their study area. The study noticed a decrease in runoff in Huifa River and attributed this decrease to both human activities and climate changes. The authors also studied variations and patterns of temperature, precipitation and runoff over a period of time. The study reveals an increasing trend in temperature and decreasing trend in runoff depth. The results showed a statistically significant increase trend in temperature and a statistically significant decrease trend in both runoff depth and runoff coefficients.

Global warming has accelerated the glacier retreat with glaciers at higher altitudes experiencing surface melting. Li et al. [42] studied the effects of glacier retreat on the water resources in eastern Xinjiang basin. They analyzed, through maps and imagery data, a total of 278 glaciers around Mount Bogda and Mount Harlik and further correlated the water shortage in eastern Xinjiang with the physical changes in the glaciers caused by retreat and recession due to global warming phenomenon. Apart from global warming, they also identified the considerable decline in local karez system's capacity, and changes in river runoff as the potential cause of retreat in glaciers.

In the global dynamics of ecology and hydrology, forest structure parameters play an important role. Based on polarization coherence tomography (PCT), Luo et al. [43] analyzed forest structure parameters from three perspectives, namely numerical simulation of Random Volume Ground model (RVoG), PolInSAR data at L-band of the test site Traunstein and Polarimetric SAR interferometry (PolInSAR) simulation of forest scene. The initial results of this research reflect high sensitivity of the vertical distribution of forest relative backscattering intensity to the forest type and density as well as polarization.

\section{Contemporary web and GIS}

There have been significant advances in the applications of GIS in conjunction with contemporary web. In this regard,
Gao et al. [44] have introduced the design and implementation of a geo-ontology-based semantic wiki system that supports users to edit and retrieve geographical knowledge. The authors first established the domain specific ontologies that consist of a set of geospatial classes, labels, and comparators as the foundation of Geo-Wiki. Geo-Wiki is implemented based on MediaWiki with pre-defined annotations derived from geo-ontology.

Semantic web technology provides leverage to access and compose multitude of services through the web. Among other types of information data, large volumes of geospatial data along with spatial data processing functions have been published on the web in the form of services. Luo et al. [45] have provided a hybrid approach to finding geospatial services on the web. Their approach matches a particular target service across four levels, namely, classification, input/ output, precondition/effect and quality of service (QoS). The approach has been effectively applied to a flood analysis case study for the Poyang Lake in China.

The advent of cloud computing gives enormous opportunities in the domain of IT research. Guo et al. [46] applied the characteristics of cloud computing with a specific focus on remote image sensing and proposed an open source remote sensing cloud platform (openRS-Cloud). The proposed platform can execute parallel remote sensing image processing applications on a massive data set in a reasonable amount of time and is very adequate for computation and data intensive remote sensing image processing applications. Experiments utilizing this platform indicate that cloud computing is a suitable choice for large-scale remote sensing image processing.

\section{Miscellaneous DE domains}

This section overviews some miscellaneous domains like geospatial navigation, distributed spatial computing, population spatialization and digital oceans.

Dynamic navigation assistance based on real-time traffic information faces several limitations including accuracy of short-term traffic forecasts in a large urban area. In this regard, experiences gained by taxi drivers can be a valuable data source for improving the quality of vehicle navigation guidance. Tang et al. [47] have carried out research in geospatial navigation and developed a vehicle navigation guidance system using taxi drivers' experience derived from the historical floating car data to formulate a new routing algorithm, named Taxi Drivers' Experience Model (TDEM). They classified road segments based on the spatiotemporal characteristics of taxi tracking data. A case study using taxi tracking data collected in Wuhan, China was presented to demonstrate the performance of this vehicle navigation system based on taxi tracking data.

As a key technique to implement Digital China, grid is an excellent and promising concept to construct a dynamic, 
inter-domain and distributed computing environment. Wu et al. [48] have presented a distributed spatial computing prototype system with the Globus Toolkit. Firstly, the architecture was proposed according to the characteristics of grid, followed by designing the spatial resource query and access interfaces for heterogeneous data sources. The authors represented an open-up hierarchical architecture for resource discovery and management to detect spatial and computing resources in grid.

Population consensus data neither are always available nor reflect the internal differences of population adequately. Zeng et al. [49] while addressing this issue proposed population spatialization technique utilizing integer night-time imagery and land use data. They applied their approach in China. Their population modeling scheme for China has a determination coefficient ranging from 0.80 to 0.95 . Their approach, as compared with contemporary efforts, reflects the population distribution more explicitly and in greater detail.

Cao et al. [50] correlated the environmental factors and birds migration with the highly pathogenic avian influenza (HPAI) by employing a logistical model based study. The authors considered environmental factors in predicting a probability map using regression. The results explain that statistically, water body proximities and national highways are relevant to the incidence of HPAI. Also, waterfowl birds are found to be important HPAI infection sources among migratory birds. The authors also studied the spatial and temporal autocorrelation of HPAI epidemics empirically. Their findings serve as a solid foundation in HPAI risk prediction.

Digital Ocean is an innovative and challenging research area in the domain of DE. The construction of the China Digital Ocean Prototype System (CDOPS) serves as a foundation for realization of Digital Ocean concept. In this regard, Zhang et al. [51] discussed the CDOPS framework, including its architectural, data and function level details. They also carried out a study on key technologies for building the 3D digital ocean environment, also focusing on techniques for visualizing digital ocean data.

The global navigation satellite system (GNSS) signals reflected by the sea surface act as a useful remote sensing tool. However, signal detection and processing are affected by the direct-path interference. In order to suppress this interference, Zhang et al. [52] have proposed an improved subspace projection algorithm. According to this algorithm, the interferential signal is at first acquired and tracked, and then a disturbance subspace is constructed according to the obtained interferential signal parameter. Interference is subsequently eliminated employing the improved subspace block projection. This method is effective as well as computationally economical. The validity of this algorithm has been proven using a series of simulation results brought about by suppressing interferences in the neighboring satellite signals and direct wave signals in a stepwise manner.
Zhang et al. [53] reviewed various approaches to calculate phase using X-ray pulsar. In doing so, they analyzed the shortcomings in the existing methodologies, such as lack of consideration of profile texture and its mathematical modeling. They based their work on the existing research, capitalizing on the Gaussian components decomposition method (GFSAP), and improved the existing methods by expressing pulsar profiles through analytical ways and establishing their relationship with phase calculation. Employing GFSAP combined with Poisson distribution, they performed profile modeling for X-ray pulsar to assess Cramér-Rao low bound (CRLB) of phase. Further, an entropy based methodology has been proposed for the estimation of profile phase rate. Zhang et al. also have demonstrated the effectiveness of their approach with specific examples.

1 Gong J Y, Xiang L G, Chen J, et al. Multi-source geospatial information integration and sharing in virtual globes. Sci China Tech Sci, 2010, 53(Suppl I): 1-6

2 Xia Y B, Pang P Y, Weng J N. Distributed GIS oriented generalized image pyramid and its practice. Sci China Tech Sci, 2010, 53(Suppl I): 99-104

3 Zhao Y, Hu C, Shen H, et al. A hierarchical organization approach of multi-dimensional remote sensing data for lightweight Web Map Services. Earth Sci Inform, 2012, 5(Suppl I): 61-75

4 Lü X F, Cheng C Q, Gong J Y, et al. Review of data storage and management technologies for massive remote sensing data. Sci China Tech Sci, 2011, 54(Suppl I): 3220-3232

5 Yang X, Weng J N, Xia Y B, et al. 3D building modeling, organization and application in digital city system. Sci China Tech Sci, 2010, 53(Suppl I): 134-142

6 Tang L, Lin L, Shao G, et al. Redefining the digital city for promoting sustainable urban development. Int J Sust Dev World Ecol, 2011, 18(Suppl I): 543-547

7 Tong D, Feng C C, Li G C, et al. Categories and characteristics of urban villages by GIS-based analysis: A case study of the Shenzhen Special Economic Zone. Sci China Tech Sci, 2010, 53(Suppl I): $150-157$

8 Zhang X D, Wang W B, Wang D F, et al. A fusion algorithm for remote sensing images based on nonsub-sampled pyramids and bidimensional empirical decomposition. Sci China Tech Sci, 2010, 53(Suppl I): 196-204

9 Nan Z T, Shu L L, Zhao Y, et al. Integrated modeling environment and a preliminary application on the Heihe River Basin, China. Sci China Tech Sci, 2011, 54(Suppl I): 2145-2156

10 Cao M L, Zhou H C, Zhang C, et al. Research and application of flood detention modeling for ponds and small reservoirs based on remote sensing data. Sci China Tech Sci, 2011, 54(Suppl I): 21382144

11 Yu J, Wu L, Zi G, et al. SDOG-based multi-scale 3D modeling and visualization on global lithosphere. Sci China Earth Sci, 2012, 55(Suppl I): 1012-1020

12 Qi Q W, Zhang A, Jiang L L, et al. Optimization of mathematical models for thematic maps. Sci China Tech Sci, 2010, 53(Suppl I): 15-24

13 Chen Z, Shen L, Zhao Y Q, et al. Parallel algorithm for real-time contouring from grid DEM on modern GPUs. Sci China Tech Sci, 2010, 53(Suppl I): 33-37

14 Dong W H, Tian Y, Zhang Y. Automatic generalization of metro maps based on dynamic segmentation. Sci China Tech Sci, 2010, 53(Suppl I): 158-165

15 Gao W X, Stein A, Gong J Y, et al. Comparing intellectual and graphical complexities of traditional and image-derived landuse maps. Sci China Tech Sci, 2010, 53(Suppl I): 205-212 
16 Sun K M, Sui H G, Li D R, et al. A new relative radiometric consistency processing method for change detection based on wavelet transform and a low-pass filter. Sci China Tech Sci, 2010, 53(Suppl I): $7-14$

17 Zhang G, Li Y, Li Z J. A new approach toward object-based change detection. Sci China Tech Sci, 2010, 53(Suppl I): 105-110

18 Yang Y, Zhou Q M, Gong J Y, et al. Gradient analysis of landscape spatial and temporal pattern changes in Beijing metropolitan area. Sci China Tech Sci, 2010, 53(Suppl I): 91-98

19 Zhou Q M, Sun B. Analysis of spatio-temporal pattern and driving force of land cover change using multi-temporal remote sensing images. Sci China Tech Sci, 2010, 53(Suppl I): 111-119

20 Weng J N, Wang Y, Cai H. Constructing a core framework of visual engine for Digital Earth system. Sci China Tech Sci, 2010, 53(Suppl I): $38-43$

21 Yue P, Gong J Y, Xiang L G, et al. Analysis-enhanced virtual globe for digital earth. Sci China Tech Sci, 2010, 53(Suppl I): 61-67

22 Lü G. Geographic analysis-oriented virtual geographic environment: Framework, structure and functions. Sci China Earth Sci, 2011, 54(Suppl I): 733-743

23 Guo H. China's Earth observing satellites for building a Digital Earth. Int J Digit Earth, 2012, 5(Suppl I): 185-188

$24 \mathrm{Xu} \mathrm{W}$. The application of China's land observation satellites within the framework of Digital Earth and its key technologies. IntJ Digit Earth, 2012, 5(Suppl I): 189-201

25 Li J R, Li H Y, Tang G, et al. Research on the strategy of angles-only relative navigation for autonomous rendezvous. Sci China Tech Sci, 2011, 54: 1865-1872

26 Li J R, Li H Y, Tang G J. Optimal multi-objective trajectory design based on close-looped control for autonomous rendezvous. Sci China Tech Sci, 2011, 54: 3091-3097

27 Xiong B, Zhang X J, Jiang W S. Semi-supervised classification based on Gaussian Mixture Model for remote imagery. Sci China Tech Sci, 2010, 53(Suppl I): 85-90

28 Xiao C C, Tian Y, Shi W Z, et al. A new method of pseudo absence data generation in landslide susceptibility mapping with a case study of Shenzhen. Sci China Tech Sci, 2010, 53(Suppl I): 75-84

29 Zhang Y, Qi L N, Yang A R, et al. Investigating spatial distribution of tourist attractions' inlinks: A case study of three mountains. Sci China Tech Sci, 2010, 53(Suppl I): 126-133

30 Gong Y X, Li G C, Liu Y, et al. Positioning localities from spatial assertions based on Voronoi neighboring. Sci China Tech Sci, 2010, 53(Suppl I): 143-149

31 Qiao Y, Liu H P, Bai M, et al. Extracting impervious surfaces from multi-source satellite imagery based on unified conceptual model by decision tree algorithm. Sci China Tech Sci, 2010, 53(Suppl I): 6874

32 Li M X, Ma Z G, Niu G Y. Modeling spatial and temporal variations in soil moisture in China. Chin Sci Bull, 2011, 56: 1809-1820

33 Jian J, Yang W, Jiang H, et al. A model for retrieving soil moisture saturation with Landsat remotely sensed data. Int J Remote Sens, 2012, 33(Suppl I): 4553-4566

34 Sheng H, Zhao H J, Huang J, et al. A spatio-velocity model based semantic event detection algorithm for traffic surveillance video. Sci China Tech Sci, 2010, 53(Suppl I): 120-125

35 Xu M, Cao C, Tong Q, et al. Remote sensing based shrub above- ground biomass and carbon storage mapping in $\mathrm{Mu}$ Us desert, China. Sci China Tech Sci, 2010, 53(Suppl I): 176-183

36 Jiang L L, Qi Q W, Zhang A, et al. Improving the accuracy of imagebased forest fire recognition and spatial positioning. Sci China Tech Sci, 2010, 53(Suppl I): 184-190

37 Tian L Q, Lu J Z, Chen X L, et al. Atmospheric correction of HJ-1A/B CCD images over Chinese coastal waters using MODISTerra aerosol data. Sci China Tech Sci, 2010, 53(Suppl I): 191-195

38 Jiang W, Zhu X, Wu J, et al. Retrieval and analysis of coal fire temperature in Wuda coalfield, Inner Mongolia, China. Chin Geogr Sci, 2011, 21(Suppl I): 159-166

39 Wang X, Guo Z, Wu L, et al. Extraction of palaeochannel information from remote sensing imagery in the east of Chaohu Lake, China. Front Earth Sci, 2012, 6(Suppl I): 75-82

40 Zhang $\mathrm{C}$, Wu J, Liu $\mathrm{H}$, et al. Imaging algorithm for synthetic aperture interferometric radiometer in near field. Sci China Tech Sci, 2011, 54(Suppl I): 2224-2231

41 Zhang A, Zhang C, Fu G, et al. Assessments of impacts of climate change and human activities on runoff with SWAT for the Huifa River Basin, northeast China. Water Resour Manage, 2012, 26(Suppl I): $2199-2217$

42 Li K M, Li Z Q, Gao W Y, et al. Recent glacial retreat and its effect on water resources in eastern Xinjiang. Chin Sci Bull, 2011, 56: 3596-3604

43 Luo H M, Li X W, Chen E X, et al. Analysis of forest backscattering characteristics based on polarization coherence tomography. Sci China Tech Sci, 2010, 53(Suppl I): 166-175

44 Gao Y, Gao S, Li R Q, et al. A semantic geographical knowledge wiki system mashed up with Google Maps. Sci China Tech Sci, 2010, 53(Suppl I): 52-60

45 Luo A, Wang Y D, Chen S H. A hybrid matching method for geospatial services in a composition-oriented environment. Sci China Tech Sci, 2010, 53(Suppl I): 213-220

46 Guo W, Gong J Y, Jiang W S, et al. OpenRS-Cloud: A remote sensing image processing platform based on cloud computing environment. Sci China Tech Sci, 2010, 53(Suppl I): 221-230

47 Tang L, Li Q, Chang X, et al. Modeling of taxi drivers' experience for routing applications. Sci China Tech Sci, 2010, 53(Suppl I): 44-51

48 Wu L, Yan M L, Gao Y, et al. A distributed spatial computing prototype system in grid environment. Sci China Tech Sci, 2010, 53(Suppl I): $25-32$

49 Zeng C, Zhou Y, Wang S, et al. Population spatialization in China based on night-time imagery and land use data. Int J Remote Sens, 2011, 32(Suppl I): 9599-9620

50 Cao C X, Xu M, Chang C Y, et al. Risk analysis for the highly pathogenic avian influenza in Mainland China using meta-modeling. Chin Sci Bull, 2010, 55: 4168-4178

51 Zhang X, Dong W, Li S, et al. China digital ocean prototype system. Int J Digit Earth, 2010, 4(Suppl I): 211-222

52 Zhang J J, Yuan H, Shuai P, et al. Research on direct-path interference suppression for bistatic radar based on GNSS reflected from the ocean surface. Sci China Tech Sci, 2010, 53(Suppl I): 3051-3055

53 Zhang H, Xu L P. An improved phase measurement method of integrated pulse profile for pulsar. Sci China Tech Sci, 2011, 54(Suppl I): 2263-2270

Open Access This article is distributed under the terms of the Creative Commons Attribution License which permits any use, distribution, and reproduction in any medium, provided the original author(s) and source are credited. 